\title{
Identification and molecular characterization of Orf virus in Argentina
}

\author{
Andrea Peralta ${ }^{1}$ - Carlos Robles ${ }^{2}$ - Agustín Martínez ${ }^{2}$ Lucía Alvarez ${ }^{1,2}$. \\ Alejandro Valera ${ }^{3} \cdot$ Gabriela Calamante $^{4} \cdot$ Guido A. König ${ }^{1,4}$
}

Received: 9 January 2015/Accepted: 5 March 2015/Published online: 22 March 2015

(C) Springer Science+Business Media New York 2015

\begin{abstract}
Orf virus (ORFV) is the etiological agent of contagious ecthyma (CE), a pustular dermatitis of sheep and goats. Outbreaks of ORFV have been observed in all geographical regions of the world, including Argentina. The origin and identity of Argentinian ORFVs are unknown, and no comparative or phylogenetic studies of these viruses have been performed. In this study, we described the sequencing and analysis of five ORFV molecular markers: a partial B2L gene (ORF011), VIR (ORF020), an envelope mature protein (ORF109), vIL10 (ORF127), and GIF (ORF117) from two particular Argentinian outbreaks of CE.
\end{abstract}

Keywords Orf virus - Contagious ecthyma ·

Parapoxvirus Phylogenetic analysis

\section{Introduction}

Orf virus (ORFV), the prototype of the genus Parapoxvirus (PPV), is the etiological agent of contagious ecthyma (CE), a severe exanthematic dermatitis that affects domestic and

Edited by William Dundon.

Andrea Peralta
peralta.andrea@inta.gob.ar

1 Consejo Nacional de Investigaciones Científicas y Técnicas (CONICET), Buenos Aires, Argentina

2 Estación Experimental Agropecuaria (EEA)-Bariloche, Instituto Nacional de Tecnología Agropecuaria (INTA), Bariloche, Río Negro, Argentina

3 Facultad de Ciencias Veterinarias, Universidad Nacional de La Plata, Buenos Aires, Argentina

4 Instituto de Biotecnología, CICVyA, INTA, Buenos Aires, Argentina wild small ruminants [1]. The disease is usually characterized by highly infectious pustules on the lips, tongue, and around the mouth [1] as well as, although less common on the feet, genitals, teats, navel, intestines, and respiratory tract $[2,3]$. The transmission occurs by direct contact or via environmental contamination $[4,5]$. A decrease in host fitness is observed, since the lesions lead to the underfeeding of young lambs $[6,7]$. CE is a zoonosis, associated with occupational activities with sheep and goats $[6,8,9]$ and in human beings consists mainly in acute skin lesions.

ORFV has a worldwide distribution, and numerous ORFV outbreaks have affected small ruminant flocks in several continents [7, 10-15]. South American ORFV outbreaks have been diagnosed by veterinarians who recognized the clinical symptoms $[16,17]$. ORFV can be distinguish from other PPV species with similar manifestations [e.g., pseudocowpoxvirus (PCPV) or bovine papular stomatitis virus (BPSV)] by genomic analyses [18, 19].

Vaccines for ORFV based on virus passaged in vivo (from scab material) or in cell-culture [20] are available. It is well known that the strains used in vaccines should be related to circulating strains to be efficacious [21, 22].

Although a commercial live attenuated ecthyma vaccine is occasionally used in some Argentinian flocks little genetic data are available regarding this vaccine [23]. Moreover, at the present, there is no information about circulating strains in this country.

PPVs possess a linear double-stranded DNA genome of 130-150 kbp with unusual high GC content (approx. $64 \%$ ) [1]. The central region of the genome contains (ORFs 009-111) genes that are present in the subfamily Chordopoxvirinae and mostly occur in a common order and orientation [1, 24], while the PPV unique and pathogenesis related genes are located in the terminal regions of the genomes [25, 26]. Particularly, the B2L gene (ORF 011; homolog of vaccinia virus F13L) encodes for a highly immunogenic 
major envelope protein of $42 \mathrm{kDa}$ [27]. This gene has been used for detection of ORFV by PCR [18] and for phylogenetic analysis of ORFV in different outbreaks [7, 13, 28, 29].

However, in the last few years, several authors have proposed the analysis of other ORFV genes in order to enrich the phylogenetic analysis. For instance virulence genes (ORF020 viral interferon resistance (VIR); ORF127 viral Interleukin-10 (vIL10) or ORF117 GM-CSF inhibitor factor (GIF)) or genes from genomic regions that may contain sufficient sequence heterogeneity to differentiate among the ORFV strains (ORF132 (VEGF); ORF109; ORF110; ORF032) [30-35].

In the present study, we sequenced and analyzed five ORFV gene markers: an internal region of B2L gene (ORF011), VIR (ORF020), a gene that codifies for an envelope mature protein (ORF109), vIL10 (ORF127) and GIF (ORF117) from two particular Argentinian outbreaks of CE.

\section{Materials and methods}

\section{Origin of samples}

In July of 2013, an exanthematic outbreak occurred in a sheep flock from Pilcaniyeu Town, Río Negro Province, Argentina $\left(41^{\circ} 07^{\prime} \mathrm{S}, 70^{\circ} 43^{\prime} \mathrm{W}\right)$. Nine Merino sheep presented wart-like lesions (dried scabs) on the lips and around the mouth. The clinical diagnosis was CE. Dried scabs were collected using scalped blades and tweezers, and the samples were stored at refrigerated temperatures until arrival to the laboratory and at $-80{ }^{\circ} \mathrm{C}$ until processing.

In addition, a historic scab sample from a sheep with CE diagnosis (Chascomús Town, Buenos Aires Province, Argentina, $35^{\circ} 34^{\prime} \mathrm{S}, 58^{\circ} 01^{\prime} \mathrm{W}$ ) collected in 1997 and preserved at $-80{ }^{\circ} \mathrm{C}$, was incorporated into our analysis.

\section{Extraction of genomic DNA}

Total DNA was extracted from the collected scabs using QIAamp DNA Mini kit (QIAGEN) according to manufactureŕs instructions. Briefly, the sample scabs were thoroughly homogenized in liquid nitrogen, and $20 \mathrm{mg}$ of the resulting tissue powder was placed in a microcentrifuge tube. Then, lysis buffer and proteinase $\mathrm{K}$ were added into microcentrifuge tube. After an incubation at $56{ }^{\circ} \mathrm{C}$ in water bath until complete tissues lysis, DNA was then extracted, according to the manufacturer's instructions, eluted with $0.2 \mathrm{~mL}$ of elution buffer and finally stored at $-20^{\circ} \mathrm{C}$.

\section{PCR reactions and sequencing of ORFV genome regions}

For ORFV detection in the samples, we used the primers $045 \mathrm{~F}$ and $045 \mathrm{R}$ following the protocol established by
Kottaridi et al. 2006 [36]. These oligonucleotides amplify an internal region from ORF045 gene (position nt 44-435).

Once the presence of viral genome was confirmed, the DNA samples were used as template for PCR reactions to amplify ORF011 (internal product), ORF020, ORF109, ORF117, and ORF127 regions as described in Table 1. The olinucleotides to amplify ORF020, ORF117 and ORF127 were designed to hybridize from ATG to Stop codon, while oligonucleotides to amplify ORF109 were designed to hybridize at $-45 \mathrm{nt}$ from ATG to $+15 \mathrm{nt}$ from Stop codon. Briefly, each reaction mixture (total volume: $50 \mu \mathrm{L}$ ) contained $10 \mu \mathrm{L} 5 \times$ GoTaq green buffer, $1 \mu \mathrm{L}$ $10 \mathrm{mM}$ dNTPs mix, $0.2 \mu \mathrm{L}$ GoTaq DNA polymerase (Promega), $100 \mathrm{ng}$ of extracted DNA, and 25 pmol of the specifics primers.

PCR products were resolved by electrophoresis in $1.2 \%$ agarose gel, stained with ethidium bromide $(10 \mathrm{mg} / \mathrm{mL})$, and then visualized under UV light.

The PCR fragments were directly sequenced in both orientations, using the amplification primers. The nucleotide (nt) sequences of the five amplicons were directly obtained with the Big Dye terminator kit TM (Applied Biosystems, Foster City, CA, USA) in an ABI $3500 \mathrm{XL}$ TM (Applied Biosystems, Foster City, CA, USA). Genomic information was derived from overlapping sequences covered by forward and reverse primers (Table 1). The sequences were annotated in the GenBank (accession numbers in Table 2).

\section{Phylogenetic analyses}

The sequences were aligned with previously published PPV sequences from GenBank (Table 2) using the Clustal $\mathrm{W}$ method, and then a manually refined alignment was performed using BioEdit software. The nucleotides corresponding to primers used to amplify target genes were removed in the alignments of sequences.

Phylogenetic trees were constructed using the Kimura-2 parameters evolution model and the neighbor-joining method with 1,000 bootstrap replicates implemented by MEGA software version 6 (Tamura, Stecher, Peterson, Filipski, and Kumar 2013).

\section{Results}

First, the clinical diagnosis of CE was assessed by PCR (ORF045 gene) in the nine samples from Pilcaniyeu 2013 outbreak (sPi13) and in the historical sample from Chascomús 1997 (sCh97). All the samples resulted positive to this analysis (data not shown), which confirmed the presence of ORFV. Then, we analyzed by PCR five different regions of interest in the genome of each sample. 


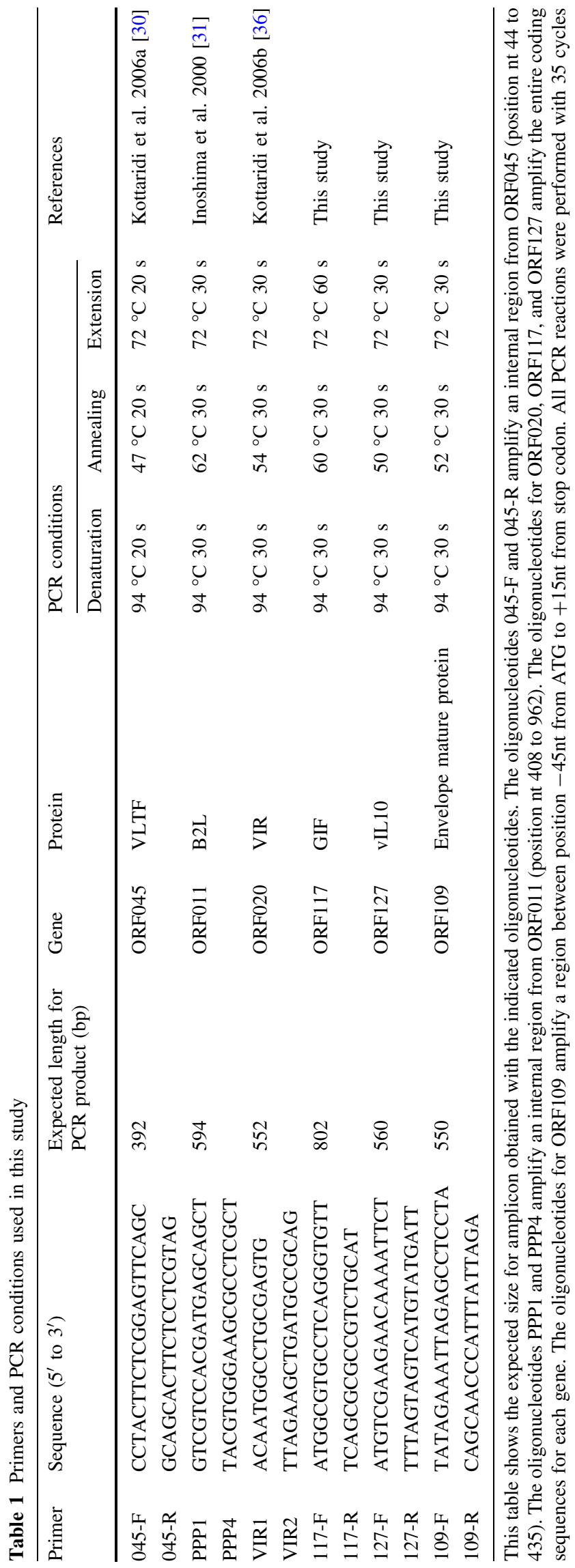

First, a specific PCR product of 594 bp from the B2L gene [18] was obtained from all nine sPi13 samples and from the $\mathrm{sCh} 97$ single sample. The nt sequence identity of this internal region of B2L gene was $100 \%$ among the nine samples from Pilcaniyeu 2013 outbreak. For this reason, all of them were treated as one single sample called sPi13.

A partial sequence of the major envelope gene of the two Argentinian outbreaks of ORFV sPi13 and sCh97 was submitted to GenBank (Table 2).

In addition, all strains sPi13, sCh97, and ORFV reference strains (IA82, NZ2 and SA00) showed a high degree of nt identity of the internal region from B2L gene (98.2-100\%). The nt identity among the Argentinian samples and Brazilian strains NE2, NE2, MT05, Orf-A, and Orf-D was between 98.8 and $99.8 \%$.

Finally, we constructed a neighbor-joining (NJ) phylogenetic tree. In this tree, sPi13 and sCH97 formed a group together with other American strains (MT05, SV29/12, SV520/11 and orf-A from Brazil; IA82, vacc03, orf-ta, orf$\mathrm{mu}$, orf-sh from USA, and three vaccine strains used in South America vaccine $A$, vaccine $B$ and vaccine $C$ ) with an intermediate bootstrap confidence value of $59 \%$ (Fig. 1a).

To improve the analysis, we also studied other ORFV genes.

Identically to the result obtained for the B2L region, in the case of the Pilcaniyeu 2013 outbreak samples, we obtained a $100 \%$ of identity for each gene analyzed in this study, thus in this case the nine samples were also treated as a single sample, sPi13.

The identity matrix of the nt sequences from VIR gene (ORF020) revealed a nucleotide identity of $96.9 \%$ between sPi13 and sCh97. This value is within the range of differences between each Argentinian strains and the reference strains (the nt identities between SPi13 and the reference strains IA82, NZ2 and SA00 were 96.6, 98.2 and $96 \%$, respectively, whereas the nt identities between sCh97 and the same reference strains were 98.6, 97.3, and $95.8 \%)$.

The NJ phylogenetic tree for the VIR sequences showed sPi13 and sCh97 shared the same phylogenetic branch. However, sPi13 grouped with NZ2 reference strain, NE2, NE1, orf-D, and orf-A Brazilian strains with an intermediate bootstrap value of $63 \%$, whereas sCh97 grouped in the same branch than IA82 reference strain with a better confidence level of $86 \%$ (Fig. 1b). The American strains could not be clustered in the same group for this gene.

When we analyzed the ORF127 (vIL10), another virulence gene, the identity between sPi13 and sCh97 was $99 \%$, which is higher than those compared to the reference strains (from 96.9 to $97.9 \%$ ). The NJ phylogenetic tree showed that the Argentinean samples are in the same branch (with high boostraps support), closer to SA00 and NZ2 reference strains than to IA82 and D1701 (Fig. 2a). 
Table 2 Published Parapoxvirus strains used for phylogenetic analyses

\begin{tabular}{|c|c|c|c|}
\hline Strain & Host & Country & Accession number \\
\hline NZ2 & Sheep & New Zealand & DQ184476 (complete genome) \\
\hline IA 82 & Sheep & USA & AY386263 (complete genome) \\
\hline SA00 & Goat & USA & AY386264 (complete genome) \\
\hline D1701 & Sheep & Germany & HM133903 (complete genome) \\
\hline VR634 (PCPV) & & New Zealand & GQ329670 (complete genome) \\
\hline BV-AR02 (BPSV) & Calf & USA & AY386265 (complete genome) \\
\hline \multicolumn{4}{|c|}{ Gene: ORF011 (B2L) } \\
\hline Orf-ta & Takin & USA & AY424971 \\
\hline Orf-mu & Musx ox & USA & AY424969 \\
\hline Orf-sh & Sheep & USA & AY424970 \\
\hline MT05 & Sheep & Brazil & JN088051 \\
\hline SV520/11 & Sheep & Brazil & JX485986 \\
\hline SV29/12 & Sheep & Brazil & JX485991 \\
\hline Orf-A & Goat & Brazil & JN613810 \\
\hline Orf-D & Sheep & Brazil & JN613809 \\
\hline NE2 & Goat & Brazil & JN088053 \\
\hline NE1 & Goat & Brazil & JN088052 \\
\hline vaccineA & Sheep & Brazil & JX485981 \\
\hline vaccineB & Sheep & Uruguay & JX485984 \\
\hline vaccineC & Sheep & Argentina & JX485978 \\
\hline vacc03 & Goat & USA & AY278209 \\
\hline ShanXi & Goat & China & JN565696 \\
\hline Nantou & Goat & Taiwan & DQ904351 \\
\hline Ind59/05 & Goat & India & DQ263304 \\
\hline Taiping & Goat & Taiwan & EU327506 \\
\hline Ind79/04 & Sheep & India & DQ263306 \\
\hline Jilin & Sheep & China & FJ808074 \\
\hline sPi13 & Sheep & Argentine & KP244324 \\
\hline sCh97 & Sheep & Argentine & KP244325 \\
\hline Reference-BPSV & NA & USA & AY424973 \\
\hline Tillquist (PCPV) & NA & USA & AY424972 \\
\hline \multicolumn{4}{|c|}{ Gene: ORF020 (VIR) } \\
\hline Orf-D & Sheep & Brazil & JN603830 \\
\hline Orf-A & Goat & Brazil & JN603831 \\
\hline Orf-ta & Takin & USA & AY424976 \\
\hline Orf-mu & Musx ox & USA & AY424974 \\
\hline Orf-sh & Sheep & USA & AY424975 \\
\hline NE1 & Goat & Brazil & JN603828 \\
\hline NE2 & Goat & Brazil & JN603829 \\
\hline Taiping & Goat & Taiwan & EU327508 \\
\hline ShanXi & Goat & China & JN565697 \\
\hline Hoping & Goat & Taiwan & EU935104 \\
\hline Nantou & Goat & Taiwan & EU327507 \\
\hline $759 / 01 \mathrm{Gr}$ & Goat & Greece & DQ275164 \\
\hline $155 / 95 \mathrm{Gr}$ & Sheep & Greece & DQ275163 \\
\hline 7389/03It & Sheep & Italy & DQ275172 \\
\hline Gansu & Sheep & China & JX391976 \\
\hline XinJiang & Sheep & China & JN565695 \\
\hline
\end{tabular}


Table 2 continued

\begin{tabular}{|c|c|c|c|}
\hline Strain & Host & Country & Accession number \\
\hline $6126 / 02$ It & Sheep & Italy & DQ275173 \\
\hline $30 / 96 \mathrm{Gr}$ & Goat & Greece & DQ275161 \\
\hline 661/95It & Sheep & Italy & DQ275169 \\
\hline sPi13 & Sheep & Argentina & KP244326 \\
\hline sCh97 & Sheep & Argentina & KP244327 \\
\hline \multicolumn{4}{|l|}{ Gene: ORF117 (GIF) } \\
\hline F09.1160S & Sheep & Finland & JF773691 \\
\hline F07.3748S & Sheep & Finland & JF773690 \\
\hline F07.808 & Reindeer & Finland & JF773686 \\
\hline B029 & Human & Germany & KF837136 \\
\hline F94.848 & Reindeer & Finland & JF773684 \\
\hline HB1 & Goat & China & KJ610834 \\
\hline SHZ1 & Goat & China & KF666567 \\
\hline SHZ2 & Goat & China & KF666566 \\
\hline SHZ3 & Goat & China & KF726847 \\
\hline Muk95/05 & Goat & India & DQ922634 \\
\hline Cam09 & Camel & India & GU460372 \\
\hline F05.990 (PCPV) & Cow & Finland & JF773682 \\
\hline F10.3081 (PCPV) & Cow & Finland & JF773683 \\
\hline sPi13 & Sheep & Argentina & KP244330 \\
\hline sCh97 & Sheep & Argentina & KP244331 \\
\hline \multicolumn{4}{|c|}{ Gene: ORF127 (vIl-10) } \\
\hline B029 & Human & Germany & KF837136 \\
\hline HB01 & Goat & China & KF990121 \\
\hline F00.120R (PCPV) & Reindeer & Finland & GQ329669 \\
\hline sPi13 & Sheep & Argentina & KP244332 \\
\hline sCh97 & Sheep & Argentina & KP244333 \\
\hline \multicolumn{4}{|l|}{ Gene: ORF109 } \\
\hline sPi13 & Sheep & Argentina & KP244328 \\
\hline sCh97 & Sheep & Argentina & KP244329 \\
\hline
\end{tabular}

$P C P V$ pseudocowpoxvirus, $B P S V$ bovine papular stomatitis virus, $N A$ not available data

The sequence analysis of ORF117 (GIF) showed a nt identity of $98.4 \%$ between sPi13 and sCh97. Both Argentinian samples showed a range of $98.5-98.8 \%$ with IA82 and NZ2 reference strains, and an identity of $96 \%$ with SA00. The phylogenetic tree revealed that $\mathrm{sPi} 13$ and sCh97 grouped in a diverse branch formed by the reference strain IA82 and NZ2, B029 German strain, and F94.848, F07.808, F07.3748S, and F09.1160S from Finland, separated from the SA00 reference strain (Fig. 2b).

The ORF109 gene encoding an envelope mature protein (EEV) has been reported as one of the most variable within the PPV genus [23, 37]. The nt identity between sPi13 and sCh97 ORF109 sequence was just $82.6 \%$. In this case, sPi13 showed a high degree of identity to IA82 (99.2\%) and sCh97 with NZ2 (99.4\%) and both pairs showed a low degree of identity to the SA00 and D1701 reference strains (less than $64 \%$ ) (Fig. 2c). Therefore, the phylogenetic analysis of ORF109 revealed that the Argentinian isolates make up a highly supported group on their own and that they are also related to the reference strains.

\section{Discussion}

This study presents the first molecular characterization of two ORFV outbreaks from Argentina.

First, we were able to confirm the presence of ORFV in all analyzed samples through the amplification of ORF045. Then we amplified an internal region of ORF011 (B2L gene, nt 388-981) that has been the main target for the 


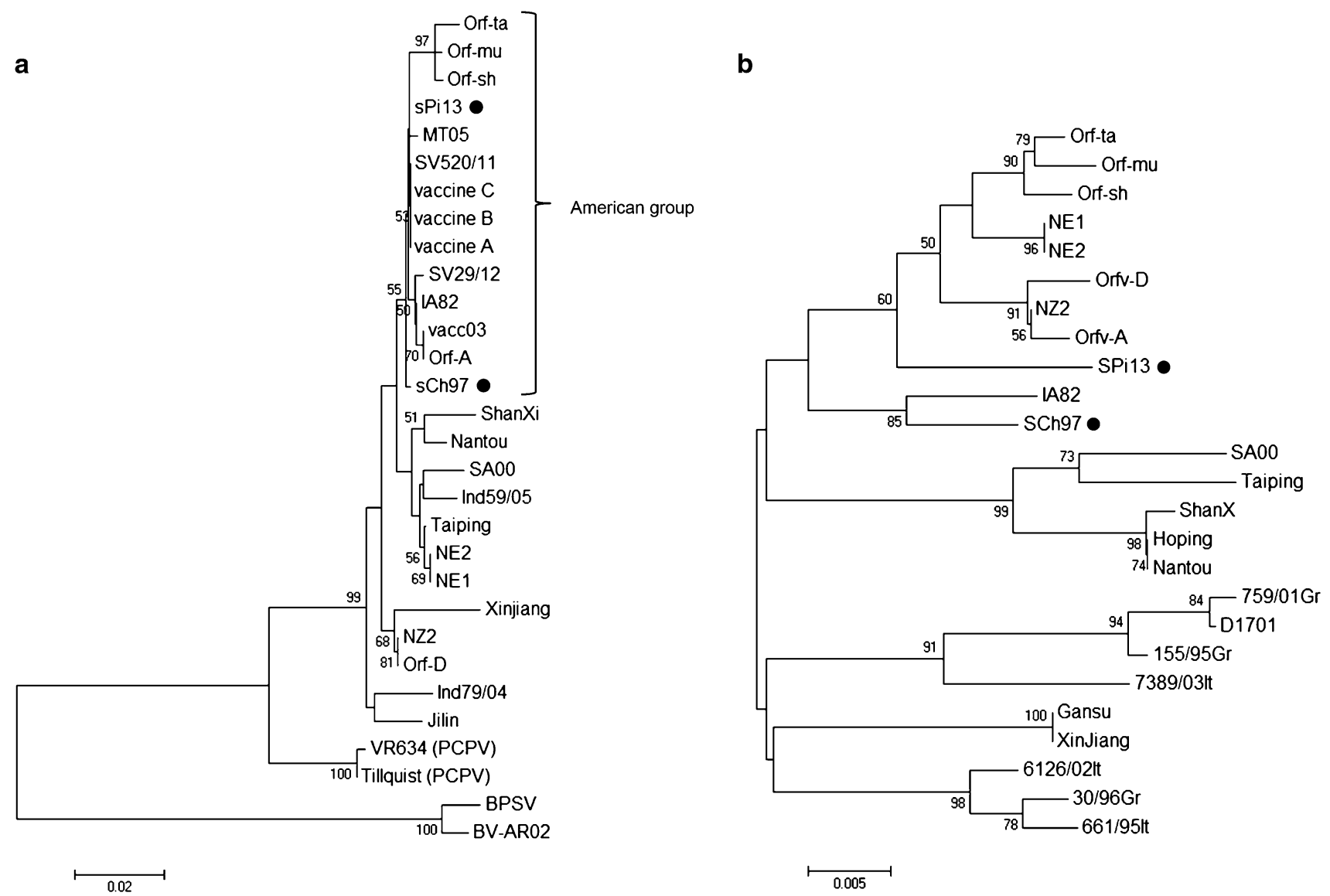

Fig. 1 Phylogenetic analyses based on nucleotide sequence of ORF011 and ORF020. Argentinian isolates are pointed with black circles. The phylogenetic relationship was constructed by the Neighbor-Joining algorithm using MEGA 6.0 software. Numbers at

phylogenetic analysis for the last years [18]. A phylogenetic analysis of this region revealed that the presence of an American cluster formed by the Brazilian, US American, and Argentinian samples (MT05, SV29/12, SV520/11, orfA; IA82, vacc03, orf-ta, orf-mu, orf-sh; sPi13 a, Ch97), along with the South American vaccine strains. The same phenomenon was observed when Schmidt et al. [23] analyzed 17 Brazilian samples and three vaccine strains available in South America (from Brazil, Argentina and Uruguay). In both cases (Schmidt's and the present study), however, the event went hand in hand with low or intermediate bootstrap values. This effect could be caused by the high level of identity observed within these samples which results in low phylogenetic signal. The nt sequence analysis confirmed the high degree of conservation of the B2L gene among ORFV from different countries and continents [13, 23, 28, 29, 37, 38].

Other researchers studied the ORF020 gene (VIR) [30, $31,33]$ since this region presents higher variability than B2L. Even when in our study we confirmed this greater nodes represent $\%$ of one thousand bootstrap replicates; only those nodes with values over $55 \%$ are shown. The scale bars are expressed in relative nucleotide sequence difference. a $\mathrm{NJ}$ tree based on ORF011. b NJ based on partial sequence of ORF020

variability (98.2-100\% for B2L vs $0.96-98.6 \%$ for VIR), we could not obtain high support values for some critical nodes.

We searched for best resolution and support in other viral genome regions in order to deepen the understanding of the phylogenetic relationship among the sequenced virus. The analysis of the virulence genes ORF117 and ORF127 showed that both Argentinian isolates belongs to the same group.

Finally, we also selected ORF109 since it is one of the most variable genes of the PPV genus according to the comparison of reference genomes [26, 39]. The analysis of this gene for the two Argentinian isolates confirmed that observation because the nt differences between them was the highest $(17.4 \%)$ of the five regions. Interestingly, for ORF109, the Argentinian strains still stayed together in the same phylogenetic group with high bootstrap value.

In summary, the two Argentinian isolates are mostly found in the same group in the analyzed genes but the level 


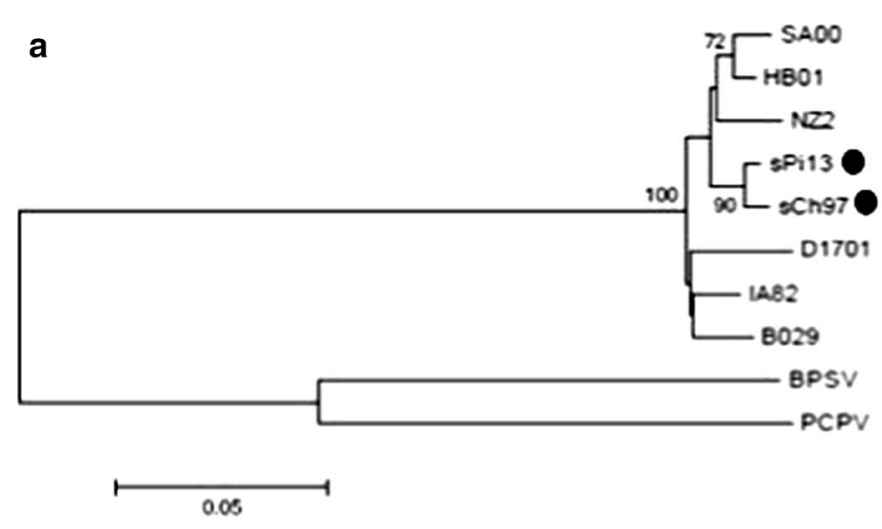

b

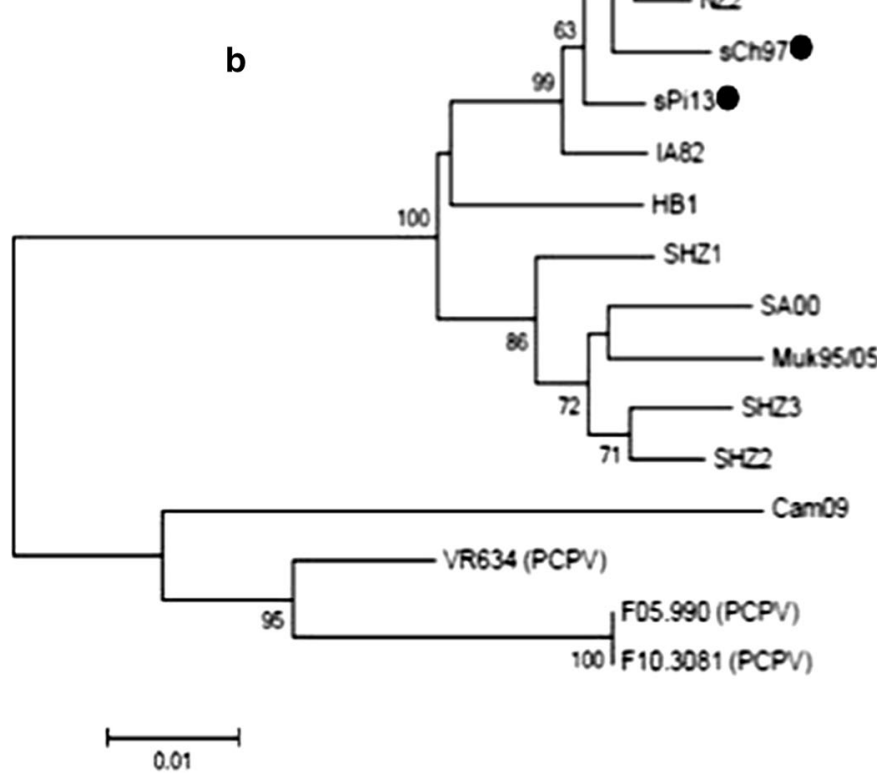

c

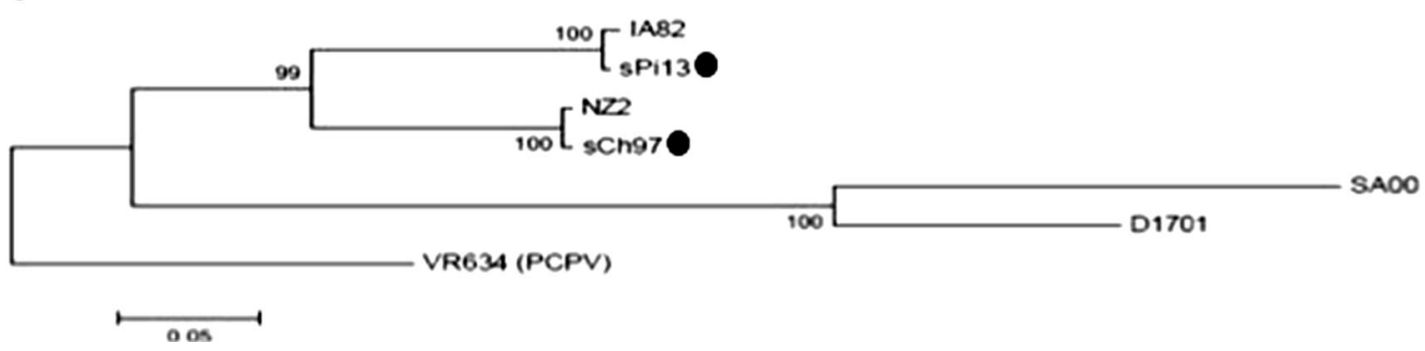

Fig. 2 Phylogenetic analyses based on the nucleotide sequences of ORF117, ORF127, and ORF109. Argentinian isolates are pointed with black circles. The phylogenetic relationship was constructed by the Neighbor-Joining algorithm using MEGA 6.0 software. Numbers

of support, the internal relationship and even some members of the group changed according to the genomic region under study.

We also propose that the study of ORF109 region could become a valuable phylogenetic tool together with multiple concatenated gene analysis, but additional at node represent $\%$ of one thousand bootstrap replicates; only those nodes with values over $55 \%$ are shown. The scale bars are expressed in relative nucleotide sequence difference. a $\mathrm{NJ}$ tree based on ORF127. b NJ tree based on ORF117. c NJ tree based on ORF109

studies are required to corroborate this hypothesis. For instance, these tools could help to differentiate strains that are identical in the usual studied gene (as the three South American vaccine strains with B2L) but different in other regions of the genome. More complete viral genome sequences or, at least, multiple gene sequencing 
studies are needed before the scientific community could properly select one or several regions to perform the phylogenetic analysis of the ORFV.

\section{References}

1. I. Damon, Chapter Poxviridae and their replication, in Fields Virology, ed. by D.M. Knipe, P.M. Howley (Raven Press Ltd, New York, 2007), pp. 2079-2081

2. M. Leite-Browning, Alabama Cooperative Extension System (2008). http://www.aces.edu/pubs/docs/U/UNP-0063/UNP-0063. pdf. Accessed 2013

3. T. Vikøren, A. Lillehaug, J. Akerstedt, T. Bretten, M. Haugum, M. Tryland, Vet. Microbiol. 127(1-2), 10-20 (2008)

4. M. Almagro, J.R. Maestre, P. Martinez, I. Malagon, E. Perez, I. Herrera, Enferm. Infecc. Microbiol. Clin. 9, 286-288 (1991)

5. D.M. Haig, A.A. Mercer, Vet. Res. 29, 311-326 (1998)

6. C. Mazur, I.I. Ferreira, F.B. Rangel, R. Galler, Vet. Microbiol. 73(4), 253-259 (2000)

7. J.S. Abrahão, R.K. Campos, G.S. Trindade, M.I. Guedes, Z.I. Lobato, C. Mazur, P.C. Ferreira, C.A. Bonjardim, E.G. Kroon, Virol J. 6, 47 (2009)

8. R. Groves, D.M. MacDonald, E.W. Jones, Arch. Dermatol. 126(12), 1649 (1990)

9. Y. Bayindir, M. Bayraktar, N. Karadag, H. Ozcan, U. Kayabas, B. Otlu, R. Durmaz, M. Doganay, New Microbiol. 34(1), 37-43 (2011)

10. Y. Inoshima, K. Murakami, D. Wu, H. Sentsui, Microbiol. Immunol. 46(8), 583-587 (2002)

11. J. Guo, Z. Zhang, J.F. Edwards, R.W. Ermel, C. Taylor Jr, A. de la Concha-Bermejillo, Virus Res. 93(2), 169-179 (2003)

12. J. Guo, J. Rasmussen, A. Wünschmann, A. de La Concha-Bermejillo, Vet. Microbiol. 99(2), 81-92 (2004)

13. M. Hosamani, V. Bhanuprakash, A. Scagliarini, R.K. Singh, Vet. Microbiol. 116(4), 317-324 (2006)

14. K.W. Chan, J.W. Lin, S.H. Lee, C.J. Liao, M.C. Tsai, W.L. Hsu, M.L. Wong, H.C. Shih, Virus Genes. 35(3), 705-712 (2007)

15. K. Zhang, Y. Shang, Y. Jin, G. Wang, H. Zheng, J. He, Z. Lu, X. Liu, Virol J. 7, 78 (2010)

16. C. Robles, F. Olaechea, in Ganadería ovina sustentable en la Patagonia Austral, (INTA Reg Patagonia Sur, Bariloche, 2001), Chapter 11, pp. 225-243.

17. C. Robles, in Diseases of Sheep (Blackwell Publishing. Chapter 71, 524-537 (2008)

18. Y. Inoshima, A. Morooka, H. Sentsui, J. Virol. Methods 84, 201-208 (2000)
19. F.J. De SantAna, F.A. Leal, R.E. Rabelo, V.A. Vulcani, C.A. Moreira Jr., J.F. Cargnelutti, E.F. Flores, J. Vet. Diagn. Invest. 25, 267-272 (2013)

20. R. Cottone, M. Buttner, B. Bauer, M. Henkel, E. Hettich, H.J. Rziha, Virus Res. 56(1), 53-67 (1998)

21. B.M. Buddle, R.W. Dellers, G.G. Schurig, Am. J. Vet. Res. 45(2), 236-263 (1984)

22. J.M. Musser, D.F. Waldron, C.A. Taylor, Am. J. Vet. Res. 73(1), 86-90 (2012)

23. C. Schmidt, J.F. Cargnelutti, M.C.S. Brum, C.K. Traesel, R Weiblen, E.F. Flores, Vet. Microbiol. 162, 245-253 (2013)

24. C. Upton, S. Slack, A.L. Hunter, A. Ehlers, R.L. Roper, J. Virol. 77(13), 7590-7600 (2003)

25. G. Delhon, E.R. Tulman, C.L. Afonso, Z. Lu, A. de la ConchaBermejillo, H.D. Lehmkuhl, M.E. Piccone, G.F. Kutish, D.L. Rock, J. Virol. 78(1), 168-177 (2004)

26. A.A. Mercer, N. Ueda, S.M. Friederichs, K. Hofmann, K.M. Fraser, T. Bateman, S. Fleming, Virus Res. 116, 146-158 (2006)

27. J.T. Sullivan, A.A. Mercer, S.B. Fleming, A.J. Robinson, Virology 202(2), 968-973 (1994)

28. I. Lojkic, Z. Cac, A. Beck, T. Bedekovic, Z. Cvetnic, B. Sostaric, Virol J. 7, 314 (2010)

29. C. Billinis, V.S. Mavrogianni, V. Spyrou, G.C. Fthenakis, Virology J. 9, 24 (2012)

30. C. Kottaridi, K. Nomikou, L. Teodori, G. Savini, R. Lelli, P. Markoulatos, O. Mangana, Vet. Microbiol. 116, 310-316 (2006)

31. Y. Inoshima, M. Ito, N. Ishiguro, J. Vet. Med. Sci. 72(6), 701-707 (2010)

32. W. Li, Z. Ning, W. Hao, D. Song, F. Gao, K. Zhao, X. Liao, M. Li, D.L. Rock, S. Luo, BMC Vet Res 8, 229 (2012)

33. J.S. Abrahão, I.A. Borges, C. Mazur, Z.I. Lobato, P.C. Ferreira, C.A. Bonjardim, G.S. Trindade, E.G. Kroon, Vet. Rec. 171(19), 476 (2013)

34. S. Friederichs, S. Krebs, H. Blum, E. Wolf, H. Lang, H. von Buttlar, M. Buttner, Virus Res. 181, 11-21 (2014)

35. H. Yang, Q. Meng, J. Qiao, Y. Peng, K. Xie, Y. Liu, H. Zhao, X. Cai, C. Chen, J. Basic Microbiol. 54, 1-6 (2014)

36. C. Kottaridi, K. Nomikou, R. Lelli, P. Markoulatos, O. Mangana, J. Virol. Methods 134, 119-124 (2006)

37. G. Venkatesan, B. Balamurugan, D.P. Bora, R. Yogisharadhya, M. Prabhu, V. Bhanuprakash, Veterinaria Italiana 47(3), 323-332 (2011)

38. K. Zhao, D. Song, W. He, H. Lu, B. Zhang, C. Li, K. Chen, F. Gao, Vet. Microbiol. 142, 408-415 (2010)

39. M. Hautaniemi, N. Ueda, J. Tuimala, A.A. Mercer, J. Lahdenpera, C.J. McInnes, J. Gen. Virol. 91, 1560-1576 (2010) 